Mathematical Research Letters 4, 791-808 (1997)

\title{
ON THE STRUCTURE OF THE STABLE NORM OF PERIODIC METRICS
}

\author{
D. Burago, S. Ivanov, and B. Kleiner
}

\begin{abstract}
We study the differentiability of the stable norm $\|\cdot\|$ associated with a $\mathbb{Z}^{n}$ periodic metric on $\mathbb{R}^{n}$. Extending one of the main results of [Ba2], we prove that if $p \in \mathbb{R}^{n}$ and the coordinates of $p$ are linearly independent over $\mathbb{Q}$, then there is a linear 2-plane $V$ containing $p$ such that the restriction of $\|\cdot\|$ to $V$ is differentiable at $p$. We construct examples where $\|\cdot\|$ it is not differentiable at a point with coordinates linearly independent over $\mathbb{Q}$.
\end{abstract}

\section{Introduction}

0.1. In this paper we study the large-scale geometry of a $\mathbb{Z}^{n}$ invariant Riemannian metric $g$ on $\mathbb{R}^{n}$. The Riemannian manifold $\left(\mathbb{R}^{n}, g\right)$ is within finite Gromov-Hausdorff distance from an $n$-dimensional normed vector space, [Bu1], which we denote $\left(\mathbb{R}^{n},\|\cdot\|\right)$. We call $\|\cdot\|$ the stable norm associated with $g$.

We denote by $B$ and $F$ the unit ball and the unit sphere of the norm $\|\cdot\|$, i.e. $B=\left\{v \in \mathbb{R}^{n}:\|v\| \leq 1\right\}, F=\left\{v \in \mathbb{R}^{n}:\|v\|=1\right\}$, respectively. Our subject is the local structure of the surface $F$, namely, the shape of its tangent cone at certain points. This is a part of the general (and wide open) question: what norms can arise as stable norms of periodic Riemannian metrics?

In [Ba2] V. Bangert - inspired by Aubry-Mather theory - made substantial progress on this question in the case of two dimensional tori (analogous results were obtained independently by F. Nazarov [Na]). [Ba2] proved that the stable norm is differentiable at every irrational point ${ }^{1}$ in $\mathbb{R}^{2}$, and that it is differentiable at a rational point only if the torus is foliated by closed geodesics representing the corresponding element of $H_{1}\left(T^{2} ; \mathbb{Z}\right) \simeq \mathbb{Z}^{2}$.

In this paper, we consider the higher dimensional case, and the regularity of the stable norm in irrational directions. The following theorem is a partial generalization of V. Bangert's result [Ba2], (see also [M4], [Ba4].)

Received August 1, 1996, Revised April 10, 1997.

The first author was supported by NSF grant DMS-95-05175.

The second author was supported by RFFR grant 96-01-00676 and CRDF grant RM1-169.

The third author was supported by NSF grants DMS-95-05175 and DMS-9626911.

${ }^{1} \mathrm{~A}$ point $p \in \mathbb{R}^{n}$ is irrational if its coordinates are linearly independent over $\mathbb{Q}$. 
Theorem 1. Let $p$ be an irrational point in $\mathbb{R}^{n}$. Then the stable norm $\|\cdot\|$ (of a $C^{3}$-smooth periodic Riemannian metric on $\mathbb{R}^{n}$ ) is differentiable in at least one nonradial direction at $p$; in other words, the tangent cone to $F$ at $\frac{p}{\|p\|}$ splits as a metric product of $\mathbb{R}$ with another cone.

Remark. Theorem 1 has a natural generalization to normal Riemannian covering spaces $\hat{M} \rightarrow M$ of a compact manifold $M$ with free abelian deck group $\mathbb{Z}^{k}$, provided $k \geq \operatorname{Dim}(M)$ (the dimension restriction is needed for the volume/packing argument given at the end of section 3 ).

The examples described in the next theorem show that the stable norm can be nondifferentiable at irrational points; hence the most optimistic attempt to generalize [Ba2] fails.

Theorem 2. For every $k$, there is an $n$ such that for almost every irrational point $p \in \mathbb{R}^{n}$, there is a $C^{k}$-smooth Riemannian metric on the $n$-torus whose stable norm is not differentiable at $p$.

It turns out that the (non)differentiability of the stable norm is directly related to the structure of certain minimizing geodesics in $\mathbb{R}^{n}$ and their interplay with a class of distance-like functions. Differentiability - or more precisely directional differentiability - correlates with the existence of curves which deviate arbitrarily far from a minimizing geodesic with bounded additional "cost". Such curves must lie in a small neighborhood of the family of minimizers associated with the point of $\mathbb{R}^{n}$; the idea behind Theorem 1 was that such curves may be constructed by joining long pieces of minimizers with short segments. We note that even for smooth metrics, the set of minimizing geodesics can be badly behaved. In the example of Theorem 2, the surface formed by the set of minimizing geodesics is highly corrugated; every curve that stays close to it must be "long".

Theorems 1 and 2 give some new information about possible singularities of the stable norm, but the picture is still far from complete. The following two questions remain open:

1. Is the finite smoothness essential for the examples in Theorem 2 , or are there similar $C^{\infty}$ examples?

2. If the stable norm is smooth and uniformly strictly convex (that is, its second fundamental form is uniformly positive definite) on an open set, is $\hat{M}$ foliated by minimizing geodesics?

The convenience of restricting ourselves to the case where $M$ is diffeomorphic to $T^{n}$ (see the remark above), and $\hat{M}$ is the universal cover of $M$ is that we may identify $\hat{M}$ with $\mathbb{R}^{n}$. Although this identification is non-invariant, the image of $Z^{n} \subset \mathbb{R}^{n}$ under this identification is. Then the stable norm $\|\cdot\|$ is given by

$$
\|v\|=\lim _{\lambda \rightarrow \infty} \frac{\rho(0, \lambda v)}{\lambda}
$$

where $\rho(\cdot, \cdot)$ is the distance function on $\hat{M} \simeq \mathbb{R}^{n}$. Sometimes this norm is also called limit norm or asymptotic norm of a metric $\rho$. The Banach space $\left(\mathbb{R}^{n},\|\cdot\|\right)$ 
approximates the metric space $\left(\mathbb{R}^{n}, \rho\right)$ in a very strong sense: there exists a constant $C=C(\rho)$ such that

$$
\forall x, y \in \mathbb{R}^{n} \quad|\|x-y\|-\rho(x, y)| \leq C
$$

(see [Bu1]). This estimate refines the statement that the Gromov-Hausdorff distance between $\left(\mathbb{R}^{n}, \rho\right)$ and $\left(\mathbb{R}^{n},\|\cdot\|\right)$ is finite.

We will use $\rho$ to denote the periodic metric under consideration and $\bar{\rho}$ for the corresponding metric on the torus $T^{n}=\mathbb{R}^{n} / \mathbb{Z}^{n}$. We denote by $U T T^{n}$ and $U T \mathbb{R}^{n}$ the unit tangent bundles for metrics $\bar{\rho}$ and $\rho$. All geodesics are parameterized by arc length. We call a geodesic $\gamma$ in $\left(T^{n}, \bar{\rho}\right)$ minimal if its lift $\tilde{\gamma}$ is minimal, i.e. if $\rho(\tilde{\gamma}(a), \tilde{\gamma}(b))=|a-b|$ for all $a, b \in \mathbb{R}$.

\section{Rotation vectors and uniformly recurrent geodesics}

1.1. We define the direction at infinity $D(\gamma) \in \mathbb{R}^{n}$ for a geodesic $\gamma: \mathbb{R} \rightarrow\left(\mathbb{R}^{n}, \rho\right)$ by

$$
D(\gamma)=\lim _{t \rightarrow \infty} \frac{\gamma(t)-\gamma(0)}{t}
$$

if the limit exists. Since $\rho(\gamma(t), \gamma(0)) \leq t$ for all $T \in \mathbb{R}$, we have

$$
\|D(\gamma)\|=\lim _{t \rightarrow \infty} \frac{\|\gamma(t)-\gamma(0)\|}{t}=\lim _{t \rightarrow \infty} \frac{\rho(\gamma(t), \gamma(0))}{t} \leq 1
$$

(the second equality follows from (1)). The rotation vector $R(\gamma) \in \mathbb{R}^{n}$ of a geodesic $\gamma: \mathbb{R} \rightarrow\left(T^{n}, \bar{\rho}\right)$ is defined by $R(\gamma)=D(\tilde{\gamma})$ where $\tilde{\gamma}$ is a lift of $\gamma$.

Recall that a geodesic $\gamma: \mathbb{R} \rightarrow\left(T^{n}, \bar{\rho}\right)$ is uniformly recurrent if for any $t_{0} \in \mathbb{R}$ the trajectory $\left\{\gamma^{\prime}(t): t \in \mathbb{R}\right\}$ visits any neighborhood of $\gamma^{\prime}\left(t_{0}\right)$ in $U T T^{n}$ with a positive frequency, that is, time average of the characteristic function of the neighborhood along the trajectory is positive. The purpose of this section is to prove the following

1.2. Proposition. If $v$ is an extreme point of $B$ (i.e. $v$ is not the midpoint of a line segment contained in $B)$, then there is a uniformly recurrent geodesic $\gamma: \mathbb{R} \rightarrow\left(T^{n}, \bar{\rho}\right)$ with $R(\gamma)=v$.

1.3. Remark. It follows from 2.4 that a geodesic $\gamma$ given by Proposition 1.2 is a minimal one. Thus we obtain a kind of existence statement for minimal geodesics with a given rotation vector. In general, not every vector $v \in F$ can be obtained as a rotation vector of a minimal geodesic. For $n \geq 3$ there exist examples of periodic metrics for which $B$ is a polyhedron and its vertices are the only possible rotation vectors of minimal geodesics (see [Ba1]).

1.4. We will prove Proposition 1.2 using the technique of minimal measures introduced by J. Mather [M2]. In fact, we only adopt the basic constructions of [M1] to our settings. 
We may also view the rotation vector of a geodesic as a function of its velocity vector, i.e. for $w \in U T T^{n}$ we set $R(w)=R(\gamma)$ where $\gamma$ is a geodesic in $\left(T^{n}, \bar{\rho}\right)$ with $\gamma^{\prime}(0)=w$. This way $R$ becomes a function defined on a subset of $U T T^{n}$. Define a map $\omega: T T^{n} \rightarrow \mathbb{R}^{n}$ as the second projection of the natural decomposition $T T^{n} \cong T^{n} \times \mathbb{R}^{n}$. The rotation vector of a geodesic $\gamma$ in $\left(T^{n}, \bar{\rho}\right)$ may be written in the form

$$
R(\gamma)=\lim _{t \rightarrow \infty} \frac{1}{t} \int_{0}^{t} \tilde{\gamma}^{\prime}=\lim _{t \rightarrow \infty} \frac{1}{t} \int_{0}^{t} \omega \circ\left(\gamma^{\prime}\right)
$$

(here $\tilde{\gamma}$ denotes a lift of $\gamma$ in $\mathbb{R}^{n}$ ). Thus the function $R$ is the average along trajectories of the function $\omega$.

Let $m$ be a finite Borel measure on $U T T^{n}$. We define its rotation vector $\mathcal{R}(m) \in \mathbb{R}^{n}$ by the formula

$$
\mathcal{R}(m)=\int_{U T T^{n}} \omega d m
$$

Clearly $\mathcal{R}(m)$ is a linear function of $m$. We call a measure $m$ on $U T T^{n}$ invariant if it is preserved by the geodesic flow of $\left(T^{n}, \bar{\rho}\right)$. If $m$ is an invariant probability measure then (by (2) and the Birkhoff ergodic theorem) the function $R$ is defined $m$-almost everywhere, and

$$
\mathcal{R}(m)=\int_{U T T^{n}} R(w) d m(w) .
$$

In particular, $\|\mathcal{R}(m)\| \leq 1$. The first observation about invariant measures is the following

1.3. Lemma. For every $v \in F$ there is an invariant probability measure $m$ with $R(m)=v$.

Proof. Let $\left(\lambda_{i}\right)$ be a sequence of positive numbers, $\lambda_{i} \rightarrow \infty$. For each $i$ let $\gamma_{i}:\left[0, l_{i}\right] \rightarrow\left(T^{n}, \bar{\rho}\right)$ be a minimal geodesic whose lift joins points 0 and $\lambda_{i} v$ in $\left(\mathbb{R}^{n}, \rho\right)$. Here $l_{i}=\rho\left(0, \lambda_{i} v\right)$. Then consider a probability measure uniformly distributed along the segment $\left[0, l_{i}\right] \subset \mathbb{R}$ and let $m_{i}$ be the image of that measure in $U T T^{n}$ under the map $t \mapsto \gamma^{\prime}(t)$. Some subsequence of $\left(m_{i}\right)$ converges weakly in the space of probability measures on $U T T^{n}$. We may assume that the original sequence $\left(m_{i}\right)$ converges to some measure $m$. It is trivial that $m$ is an invariant measure. Then

$$
\mathcal{R}(m)=\int_{U T T^{n}} \omega d m=\lim \int_{U T T^{n}} \omega d m_{i}=\lim \frac{\lambda_{i} v}{l_{i}}=\frac{v}{\|v\|}
$$

(the last equality follows from the definition of the stable norm). Since $\|v\|=1$, the lemma follows. 
1.4. Now suppose that $v$ is an extreme point of $B$ (i.e. no line segment contained in $B$ has its midpoint at $v$ ). Let $\mathcal{M}(v)$ denote the set of all invariant probability measures $m$ with $\mathcal{R}(m)=v$. This set is convex and compact (with respect to the weak topology). By the Krein-Milman theorem there is a measure $m$ which is an extreme point of $\mathcal{M}(v)$. Such a measure $m$ is ergodic with respect to the geodesic flow. Indeed, if $0<\lambda<1$ and $m=\lambda m_{1}+(1-\lambda) m_{2}$ for some invariant probability measures $m_{1}$ and $m_{2}$, then $v=\mathcal{R}(m)=\lambda \mathcal{R}\left(m_{1}\right)+(1-\lambda) \mathcal{R}\left(m_{2}\right)$, so the extremeness of $v$ in $B$ implies that $\mathcal{R}\left(m_{1}\right)=\mathcal{R}\left(m_{2}\right)=v$. Since $m$ is an extreme point of $\mathcal{M}(v)$ it follows that $m_{1}=m_{2}$.

Let $U$ be an open subset of $U T T^{n}$ with $m(U)>0$. The ergodicity of $m$ implies that $m$-almost every trajectory of the geodesic flow visits $U$ with positive frequency. Since the topology of $U T T^{n}$ has a countable base, $m$-almost every trajectory does this for all open sets of positive measure. Note that $m$-almost every trajectory is contained in $\operatorname{supp}(m)$, the support of measure $m$. Since for an open $U \subset U T T^{n}$ the condition $U \cap \operatorname{supp}(m) \neq \varnothing$ implies $m(U)>0$, it follows that $m$-almost every trajectory is uniformly recurrent. On the other hand, $R(w)=\mathcal{R}(m)=v$ for $m$-almost all $w \in U T T^{n}$ since $m$ is ergodic. This completes the proof of Proposition 1.2.

\section{Generalized coordinates and minimizers}

2.1. If $L: \mathbb{R}^{n} \rightarrow \mathbb{R}$ is a linear function we let $\|L\|$ denote its norm in the space $\left(\mathbb{R}^{n},\|\cdot\|\right)^{*}$, i.e. $\|L\|=\max \{\mid L(x):\|x\|=1\}$. We say that a linear function $L$ supports $B$ at a point $v \in F$ if $\|L\|=1$ and $L(v)=1$. Geometrically it means that a hyperplane $L^{-1}(1)$ touches the surface $F$ at $p$. We denote by $F^{*}(v)$ the set of linear functions supporting $B$ at $v$, and by $F^{*}$ the set of all linear functions $L$ with $\|L\|=1$.

Let $L \in F^{*}$ and let $f$ be a real-valued function on $\left(\mathbb{R}^{n}, \rho\right)$. We say that $f$ is a generalized coordinate associated with $L$ if

(2.1.1) $f$ is a 1-Lipschitz function with respect to $\rho$, i.e. $|f(x)-f(y)| \leq \rho(x, y)$ for all $x, y \in \mathbb{R}^{n}$.

(2.1.2) The function $(f-L)$ is $\mathbb{Z}^{n}$-periodic, i.e. for every $x \in \mathbb{R}^{n}$ and $h \in \mathbb{Z}^{n}$ we have $f(x+h)=f(x)+L(h)$.

Such function were used in [Bul2] to make volume estimates. We extract a construction from [Bul2] in the following statement.

2.2. Proposition. For any $L \in F^{*}$ there is a generalized coordinate associated with $L$.

Proof. Define a function $f$ on $\mathbb{R}^{n}$ by

$$
f(x)=\limsup _{\|y\| \rightarrow \infty}(L(y)-\rho(x, y)) .
$$

First, we need to prove that all values of $f$ are finite. This follows from (1) and the equality

$$
\limsup _{\|y\| \rightarrow \infty}(L(y)-\|x-y\|)=L(x)
$$


which holds since $\|L\|=1$. Then $f$ is 1-Lipschitz as it is the supremum of a family of 1-Lipschitz functions. Finally, for any $x \in \mathbb{R}^{n}$ and $h \in \mathbb{Z}^{n}$ we have

$$
\begin{aligned}
f(x+h) & =\limsup _{\|y\| \rightarrow \infty}(L(y)-\rho(x+h, y)) \\
& =\limsup _{\|y-h\| \rightarrow \infty}(L(y-h)-\rho(x, y-h))+L(h)=f(x)+L(h),
\end{aligned}
$$

so $f$ satisfies (2.1.2).

2.3. Let $f$ be a generalized coordinate and let $\gamma: \mathbb{R} \rightarrow\left(\mathbb{R}^{n}, \rho\right)$ be a geodesic. We say that $\gamma$ is calibrated by $f$, or that $\gamma$ is an $f$-calibrated, if

$$
f(\gamma(b))-f(\gamma(a))=b-a
$$

for all $a, b \in \mathbb{R}$.

Note that for an arbitrary unit-speed curve $\gamma$ in $\left(\mathbb{R}^{n}, \rho\right)$ we have

$$
f(\gamma(b))-f(\gamma(a)) \leq \rho(\gamma(a), \gamma(b)) \leq|b-a|
$$

for all $a, b \in \mathbb{R}$. Therefore if $\gamma$ is $f$-calibrated then $\gamma$ is a minimal geodesic. It is clear that being $f$-calibrated is actually a local property: if $\gamma$ is not $f$-calibrated then for any $\varepsilon>0$ there exists $a \in \mathbb{R}$ such that (3) fails for $[a, b]=[a, a+\varepsilon]$. From (2.1.2) it follows that any integer translate of an $f$-calibrated geodesic is also an $f$-calibrated.

2.4. Proposition. Let $\gamma: \mathbb{R} \rightarrow\left(\mathbb{R}^{n}, \rho\right)$ be a lift of a uniformly recurrent geodesic with $D(\gamma)=v \in F$, and $L \in F^{*}(v)$. Then $\gamma$ is calibrated by any generalized coordinate associated with $L$.

Proof. Let $f$ be a generalized coordinate associated with $L$, and suppose that $\gamma$ is not $f$-calibrated. We may assume that (3) fails for $a=0$ and $b=1$, say $f(\gamma(1))-f(\gamma(0))=1-\varepsilon$ where $\varepsilon>0$. Let $U$ be a neighborhood of $\gamma^{\prime}(0)$ in $U T \mathbb{R}^{n}$ such that for every geodesic $\gamma_{1}$ in $\left(\mathbb{R}^{n}, \rho\right)$ with $\gamma_{1}^{\prime}(0) \in U$ we have $\rho\left(\gamma_{1}(t), \gamma(t)\right)<\varepsilon / 3$ for all $t \in[0,1]$. Since $\gamma$ is a lift of a uniformly recurrent geodesic, there exists a sequence $\left(t_{i}\right)_{i=1}^{\infty}$ of real numbers such that for all $i$ we have $t_{i+1} \geq t_{i}+1, t_{i} / i \leq T_{0}<\infty$, and $\gamma^{\prime}\left(t_{i}\right) \in U+h_{i}$ for some $h_{i} \in \mathbb{Z}^{n}$. By (2.1.2) and the choice of $U$ we have

$$
f\left(\gamma\left(t_{i}+1\right)\right)-f\left(\gamma\left(t_{i}\right)\right) \leq 1-\varepsilon / 3
$$

for each $i$. Summing up these inequalities for $i=1, \ldots, m$, together with obvious ones

$$
f\left(\gamma\left(t_{i+1}\right)\right)-f\left(\gamma\left(t_{i}\right)\right) \leq t_{i+1}-t_{i}
$$

we obtain that

$$
f\left(\gamma\left(t_{m}+1\right)\right)-f(\gamma(0)) \leq t_{m}+1-\frac{m \varepsilon}{3} \leq\left(1-\frac{\varepsilon}{3 T_{0}}\right) t_{m}+1
$$


Therefore

$$
\limsup _{t \rightarrow \infty} \frac{f(\gamma(t))-f(\gamma(0))}{t} \leq 1-\frac{\varepsilon}{3 T_{0}}<1 .
$$

Since $|f-L|$ is bounded, one may replace $f$ by $L$ in this estimate. On the other hand,

$$
\lim _{t \rightarrow \infty} \frac{L(\gamma(t))-L(\gamma(0))}{t}=L(D(\gamma))=L(v)=1
$$

(the first equality follows from the definition of $D(\gamma)$, see 1.1). This contradiction proves the proposition.

We will apply Proposition 2.4 at a point $v \in F$ where $B$ has several different supporting lienar functions (this may happen if $F$ is not smooth at $v$ ), so the same geodesic $\gamma$ will be calibrated by the generalized coordinates associated with each of these functions. The following Proposition 2.5 tells us that in this case all the generalized coordinates calibrating $\gamma$ have similar local behavior near $\gamma$.

2.5 Proposition. Let $\rho$ be a $C^{3}$ metric. Then there is a constant $C=C(\rho)$ with the following property. If a geodesic $\gamma$ is calibrated by two generalized coordinates $f_{1}$ and $f_{2}$ (possibly associated with different linear functions), then for $d=f_{1}(\gamma(0))-f_{2}(\gamma(0))$ the inequality

$$
\left|f_{1}(x)-f_{2}(x)-d\right| \leq C \cdot \rho(x, \gamma)^{2}
$$

holds for all $x \in \mathbb{R}^{n}$. Here $\rho(x, \gamma)$ denotes $\inf \{\rho(x, \gamma(t)): t \in \mathbb{R}\}$.

Proof. It suffices to prove the statement only in the case $d=0$ (just add a constant to $f_{2}$ ). Pick constants $\varepsilon>0$ and $r>\varepsilon$ such that $r+\varepsilon$ is less than the injectivity radius of $\left(\mathbb{R}^{n}, \rho\right)$. Fix $t \in \mathbb{R}$ and denote $c=f_{1}(\gamma(t))=f_{2}(\gamma(t))$. Since $f_{1}$ and $f_{2}$ are 1-Lipschitz functions, we have

$$
f_{i}(\gamma(t+r))-\rho(\gamma(t+r), x) \leq f_{i}(x) \leq f_{i}(\gamma(t-r))+\rho(\gamma(t-r), x),
$$

or

$$
f_{\text {low }}(x):=c+r-\rho(\gamma(t+r), x) \leq f_{i}(x) \leq c-r+\rho(\gamma(t-r), x):=f_{\text {up }}(x)
$$

for any $x \in \mathbb{R}^{n}, i=1,2$. Both $f_{\text {low }}$ and $f_{\text {up }}$ are $C^{2}$-smooth functions of $x$ within $\varepsilon$-neighborhood of $\gamma(t)$, and their values and first derivatives at $\gamma(t)$ coincide. (The values are equal to $c$ and the gradients are equal to $\gamma^{\prime}(t)$ ). So if $C>2 / \varepsilon$ and $C$ is an upper bound for the second derivative of a function $\rho\left(x_{0}, \cdot\right)$ between its values $r-\varepsilon$ and $r+\varepsilon$, then

$$
\left|f_{1}(x)-f_{2}(x)\right| \leq f_{\text {up }}(x)-f_{\text {low }}(x) \leq C \cdot \rho(x, \gamma(t))^{2}
$$

Since $t$ is arbitrary, the proposition follows. 


\section{A tangent cone of $B$ at an irrational point}

3.1. We call a vector $v \in \mathbb{R}^{n}$ irrational if its coordinates are linearly independent over $\mathbb{Q}: v$ is not an irrational vector if and only if there is a nonzero linear function $L \in\left(\mathbb{Q}^{n}\right)^{*} \subset\left(\mathbb{R}^{n}\right)^{*}$ such that $L(v)=0$. The latter description implies that non-irrational vectors form a set of zero measure in $\mathbb{R}^{n}$ since this set is a union of countably many hyperplanes. If $v$ is an irrational vector then $\lambda v$ is irrational for any $\lambda \in \mathbb{R}$.

For $v \in \mathbb{R}^{n}$ we denote by $\langle v\rangle$ the line $\{\lambda v: \lambda \in \mathbb{R}\} \subset \mathbb{R}^{n}$. If $v$ is an irrational vector the union of lines $\langle v\rangle+\mathbb{Z}^{n}:=\bigcup_{h \in \mathbb{Z}^{n}}(\langle v\rangle+h)$ is dense in $\mathbb{R}^{n}$.

3.2. For $v \in F$ we denote by $C_{v}(B)$ the tangent cone of $B$ at $v$. We define a tangent cone as an intersection of half-spaces

$$
C_{v}(B)=\bigcap_{L \in F^{*}(v)}\left\{x \in \mathbb{R}^{n}: L(x) \leq 0\right\}
$$

A convex cone is called sharp if it contains no straight lines. From (4) it follows that $C_{v}(B)$ is sharp if and only if $F^{*}(v)$ contains $n$ linearly independent linear functions. If $C_{v}(B)$ is sharp then obviously $v$ is an extreme point of $B$.

3.3. Theorem. Let $\rho$ be a $C^{3}$-smooth periodic Riemannian metric on $\mathbb{R}^{n}$, let $B$ be the unit ball of its stable norm, and let $v$ be an irrational vector in the boundary of $B$. Then the tangent cone $C_{v}(B)$ is not sharp.

Proof. Suppose that $C_{v}(B)$ is a sharp cone. By Proposition 1.5 there exists a geodesic $\gamma: \mathbb{R} \rightarrow\left(\mathbb{R}^{n}, \rho\right)$ with $D(\gamma)=v$ which is a lift of a uniformly recurrent geodesic. Pick $n$ linearly independent functions $L_{1}, \ldots, L_{n}$ from $F^{*}(v)$. For each $i=1, \ldots, n$ construct a generalized coordinate $f_{i}$ associated with $L_{i}$. We may assume that $f_{i}(\gamma(0))=0$ and so $f_{i}(\gamma(t))=t$ for all $t \in \mathbb{R}$. Define a map $\bar{f}:\left(\mathbb{R}^{n}, \rho\right) \rightarrow \mathbb{R}^{n-1}$ by

$$
\bar{f}=\left(f_{1}-f_{2}, f_{2}-f_{3}, \ldots, f_{n-1}-f_{n}\right) .
$$

For any $x \in \mathbb{R}^{n}$ and $h \in \mathbb{Z}^{n}$ we have

$$
\bar{f}(x+h)=\bar{f}(x)+\bar{L}(h)
$$

where the linear map $\bar{L}: \mathbb{R}^{n} \rightarrow \mathbb{R}^{n-1}$ is defined by

$$
\bar{L}=\left(L_{1}-L_{2}, L_{2}-L_{3}, \ldots, L_{n-1}-L_{n}\right) .
$$

Therefore for any $h \in \mathbb{Z}^{n}$ the values of $\bar{f}$ are equal to $\bar{L}(h)$ along the geodesic $\gamma+h$, so we have the estimate

$$
|\bar{f}(x)-\bar{L}(h)| \leq C \cdot \rho(x, \gamma+h)^{2}
$$


(see 2.4 and 2.5). Applying this for points $x$ of another geodesic $\gamma+h_{1}$ we obtain that

$$
\left|\bar{L}\left(h_{1}\right)-\bar{L}(h)\right| \leq C \cdot \rho\left(\gamma+h_{1}, \gamma+h\right)^{2}
$$

for all $h, h_{1} \in \mathbb{Z}^{n}$. (For two geodesics $\gamma_{1}$ and $\gamma_{2}$ we denote by $\rho\left(\gamma_{1}, \gamma_{2}\right)$ the distance between them as subsets of $\left.\left(\mathbb{R}^{n}, \rho\right)\right)$. Therefore

$$
\rho\left(\gamma+h_{1}, \gamma+h\right) \geq \sqrt{\left|\bar{L}\left(h_{1}\right)-\bar{L}(h)\right| / C}
$$

for all $h, h_{1} \in \mathbb{Z}^{n}$.

The functions $f_{i}-L_{i}$ are bounded and $f_{i}(\gamma(t))=t$ for $t \in \mathbb{R}$, so $\left|L_{i}(\gamma(t))-t\right| \leq$ $C$ for some constant $C$ not depending on $t$. Hence there is an $R>0$ such that $|\gamma(t)-v t| \leq R$ for all $t \in \mathbb{R}$ (this follows from the fact that $v t$ is the only point of $\mathbb{R}^{n}$ at which the values of functions $L_{i}$ are all equal to $t$ ). Thus any straight line of the form $\langle v\rangle+h$ is contained within $R$-neighborhood of the corresponding geodesic $\gamma+h$.

Note that the map $\bar{L}$ is surjective and $\operatorname{Ker} \bar{L}=\langle v\rangle$. Let $U$ be a bounded neighborhood of a unit cube in $\mathbb{R}^{n-1}, U_{1}$ be a bounded set in $\mathbb{R}^{n}$ such that $U \subset L\left(U_{1}\right)$, and $U_{2}$ be the $R$-neighborhood of $U_{2}$. Since $\langle v\rangle+\mathbb{Z}^{n}$ is dense in $\mathbb{R}^{n}$ and $\bar{L}(v)=0$, the set $\bar{L}\left(\mathbb{Z}^{n}\right)$ is dense in $\mathbb{R}^{n-1}$. For a small $\varepsilon>0$ one can find a collection of points $y_{1}, \ldots, y_{N} \in U \cap \bar{L}\left(\mathbb{Z}^{n}\right)$ such that $N \geq(1 / \varepsilon)^{n-1}$ and $\left|y_{i}-y_{j}\right|>\varepsilon$ for $i \neq j$. Let $y_{i}=\bar{L}\left(h_{i}\right)$ where $h_{i} \in \mathbb{Z}^{n}$. Consider the geodesics $\gamma+h_{i}$ in $\left(\mathbb{R}^{n}, \rho\right)$. They all cross the region $U_{2}$. If $U_{3}$ is the 1-neighborhood of $U_{2}$ in $\left(\mathbb{R}^{n}, \rho\right)$, then the intersection of each of our geodesics $\gamma+h_{i}$ with $U_{3}$ will contain an interval of length 2 inside $U_{3}$.

By (5), the pairwise distances between the geodesics $\gamma+h_{i}$ are not less than $\sqrt{\varepsilon / C}$. Hence their tubular neighborhoods of radius $\sqrt{\varepsilon / 4 C}$ are disjoint. Summing up Riemannian volumes of these tubular neighborhoods we obtain a lower bound for the volume of $U_{3}$ :

$$
\operatorname{Vol}\left(U_{3}\right) \geq(1 / \varepsilon)^{n} \cdot(\varepsilon / 4 C)^{(n-1) / 2}=c_{1} \varepsilon^{-(n+1) / 2}
$$

for some $c_{1}>0$. Since $\varepsilon$ is arbitrarily small, it follows that $\operatorname{Vol}\left(U_{3}\right)=\infty$. This is impossible since $U_{3}$ is bounded.

\section{An example}

In this section we construct the examples described in Theorem 2. The idea of the construction is to begin by producing a set of minimizers. This set will be (the closure of) a highly corrugated surface. The estimate of lemma (5), together with elementary geometric considerations, suggests that the cross-section of this surface may be regarded as a curve which stretches distance in the following very strong sense: the distance between the images is at least the square root of the distance between pre-images modulo 1 . On the other hand, this curve has to have irrational rotation vector, and it has to be invariant under a group of 
diffeomorphisms. We construct this curve as a sum of two curves. One of them is a small periodic curve ( with rotation vector zero), which stretches distance and persists under a group of diffeomorphisms having dense orbits on the curve. A construction of a curve analogous properties was used in [Bul3]. The other one is a smooth curve with irrational rotation vector which lies in a submanifold of high codimension. The first curve will be constructed using trigonometric series, where the following approximation condition is a technical requirement used to estimate the denominators in the coefficients of the series.

We call a number $\alpha \in \mathbb{R}$ approximable if for any $\varepsilon>0$ there exists a sequence of rational numbers $\left\{p_{k} / q_{k}\right\}_{k=1}^{\infty}$ such that

$$
\left|\alpha-p_{k} / q_{k}\right|<1 / q_{k}^{2}
$$

and

$$
q_{k}^{1+\varepsilon / 2}<q_{k+1}<\frac{1}{2} q_{k}^{1+\varepsilon}
$$

for all $k \geq 1$.

4.1. Lemma. Almost all real numbers are approximable.

Proof. For a given $\alpha \in \mathbb{R}$, every convergent $p_{k} / q_{k}$ of the corresponding continued fraction satisfies $\left|\alpha-p_{k} / q_{k}\right|<1 / q_{k}^{2}$. By Khinchin's theorem, it is known (e. g. $[\mathrm{Ka}])$ that for almost every $\alpha$ the denominators of these convergents grow exponentially: the $\operatorname{limit} \lim _{k \rightarrow \infty}\left(\log q_{k}\right) / k$ exists. For such $\alpha$, it is certainly possible to find a subsequence of $\left\{q_{k}\right\}$ to satisfy (6).

Let $\gamma: \mathbb{R} \rightarrow \mathbb{R}^{n}$ be a continuous curve and $r>1$ be an integer. We say that $\gamma$ is $r$-stretching if there exists $\delta>0$ such that $|\gamma(x)-\gamma(y)| \geq|x-y|^{1 / r}$ whenever $|x-y| \leq \delta$.

4.2. Proposition. Let $r>1$ be an integer, and let $\alpha \in \mathbb{R}$ be approximable. Then there exists an $r$-stretching 1-periodic curve $\gamma: \mathbb{R} \rightarrow \mathbb{R}^{8 r}$ such that the function

$$
x \mapsto \gamma(x+\alpha)-\gamma(x)
$$

is $C^{1}$-smooth.

Proof. Let $\varepsilon=1 / 2(r-1)$ and pick a sequence $\left\{p_{k} / q_{k}\right\}$ as in 4.1. We assume that $q_{1}>10^{4 r(r-1)}$ which, by (6), implies that $q_{k+1}>10^{r} q_{k}$ for all $k$.

We identify $\mathbb{R}^{8 r}$ with $\mathbb{C}^{4 r}$ and use the notation $E(t)=\exp (2 \pi i t) \in \mathbb{C}$ for $t \in \mathbb{R}$. Let $v_{k}$ be the $k^{t h}$ basis vector of $\mathbb{C}^{4 r}$ for $1 \leq k \leq 4 r$, and $v_{k+4 r}=v_{k}$ for all $k \in \mathbb{Z}$. Define $\gamma: \mathbb{R} \rightarrow \mathbb{C}^{4 n}$ by

$$
\gamma(x)=\sum_{k=1}^{\infty} q_{k}^{-1 / 2 r} E\left(q_{k} x\right) v_{k} .
$$


Then

$$
\gamma(x+\alpha)-\gamma(x)=\sum_{k=1}^{\infty} q_{k}^{-1 / 2 r} E\left(q_{k} \alpha\right) E\left(q_{k} x\right) v_{k} .
$$

The formal derivative of these series has the form

$$
\sum_{k=1}^{\infty} q_{k}^{1-1 / 2 r} E\left(q_{k} \alpha\right) E\left(q_{k} x\right) v_{k} .
$$

Since $q_{k} \alpha-p_{k}<1 / q_{k}$, we have $E\left(q_{k} \alpha\right)<2 \pi q_{k}^{-1}$, so this formal derivative converges absolutely. It follows that the function $x \mapsto \gamma(x+\alpha)-\gamma(x)$ is $C^{1}$.

We will prove that $|\gamma(x)-\gamma(y)| \geq|x-y|^{1 / r}$ whenever $|x-y|<1 / 2 q_{1}$. Assume that $x<y<x+1 / 2 q_{1}$ and denote $\delta=y-x$. We have

$$
\gamma(y)-\gamma(x)=\sum_{k=1}^{\infty} q_{k}^{-1 / 2 r} E\left(q_{k} x\right)\left(E\left(q_{k} \delta\right)-1\right) v_{k} .
$$

The right inequality in (6) implies $\frac{1}{2} q_{k+1}^{-1}>q_{k}^{-1-\varepsilon}$, so the intervals $\left(q_{k}^{-1-\varepsilon}, \frac{1}{2} q_{k}^{-1}\right)$ cover the interval $\left(0,1 / 2 q_{1}\right)$. Pick an index $m$ for which $\delta \in\left(q_{m}^{-1-\varepsilon}, \frac{1}{2} q_{m}^{-1}\right)$. For every integer $j>-m / 4 r$ denote $a_{j}=q_{m+4 r j}$ and set $a_{j}=0$ for $j \leq-m / 4 r$. We have $\delta \in\left(a_{0}^{-1-\varepsilon}, \frac{1}{2} a_{0}^{-1}\right)$, so

$$
\delta^{-1 /(1+\varepsilon)}<a_{0}<\frac{1}{2} \delta^{-1}
$$

and, by (6),

$$
a_{j+1}>a_{j}^{(1+\varepsilon / 2)^{4 r}}>a_{j}^{1+2 r \varepsilon}=a_{j}^{2+2 \varepsilon} .
$$

It follows that $a_{j+1} \geq 10^{2 r} a_{j}$ for all $j$, and

$$
a_{1}>a_{0}^{2+2 \varepsilon}>\delta^{-(2+2 \varepsilon) /(1+\varepsilon)}=\delta^{-2}, \quad a_{-1}<a_{0}^{1 /(2+2 \varepsilon)}<a_{0}^{1 / 2}<\delta^{-1 / 2} .
$$

The $m$ th coordinate of the vector $\gamma(y)-\gamma(x)$ can be written as

$$
\sum_{j \in \mathbb{Z}} a_{j}^{-1 / 2 r} E\left(a_{j} x\right)\left(E\left(a_{j} \delta\right)-1\right)
$$

So

$$
|\gamma(y)-\gamma(x)| \geq a_{0}^{-1 / 2 r}\left|E\left(a_{0} \delta\right)-1\right|-\sum_{j \neq 0} a_{j}^{-1 / 2 r}\left|E\left(a_{j} \delta\right)-1\right|
$$

Since $|E(t)-1| \geq 4 t$ for $0 \leq t \leq 1 / 2$, we have

$$
a_{0}^{-1 / 2 r}\left|E\left(a_{0} \delta\right)-1\right| \geq 4 \delta a_{0}^{1-1 / 2 r}>4 \delta^{1-(1-1 / 2 r) /(1+\varepsilon)}=4 \delta^{1 / r} .
$$


For $j>0$ we have $a_{j}>10^{2 r(j-1)} a_{1}>10^{2 r(j-1)} \delta^{-2}$, so

$$
\begin{aligned}
\sum_{j>0} a_{j}^{-1 / 2 r}\left|E\left(a_{j} \delta\right)-1\right| & \leq \sum_{j>0} 2 a_{j}^{-1 / 2 r}<\sum_{j>0} 2\left(10^{2 r(j-1)} \delta^{-2}\right)^{-1 / 2 r} \\
& =\sum_{j>0} 2 \cdot 10^{-j+1} \delta^{1 / r}<2.5 \delta^{1 / r}
\end{aligned}
$$

For $-m / 4<j<0$ we have $10^{4 r}<a_{j}<10^{j+1} a_{-1}<10^{j+1} \delta^{-1 / 2}$, so

$$
\begin{aligned}
\sum_{j<0} a_{j}^{-1 / 2 r}\left|E\left(a_{j} \delta\right)-1\right| & \leq \sum_{j<0} 10^{-2} \cdot 2 \pi a_{j} \delta \leq \sum_{j<0} 10^{-1}\left(10^{j+1} \delta^{-1 / 2}\right) \delta \\
& =\sum_{j<0} 10^{j} \delta^{1 / 2}<0.5 \delta^{1 / 2} \leq 0.5 \delta^{1 / r} .
\end{aligned}
$$

It follows that $|\gamma(x)-\gamma(y)| \leq(4-2.5-0.5) \delta^{1 / r}=\delta^{1 / r}$.

4.3. Proposition. For every integer $n \geq 7$ and $v \in \mathbb{R}^{n}$ there is a threedimensional smooth submanifold $M \subset T^{n}$ and a smooth flow on $M$, one of whose trajectories has the rotation vector $v$ (as a curve in $T^{n}$ ).

Proof. Let $M_{0}$ be a two-dimensional orientable surface of genus $n$ equipped with a negatively curved Riemannian metric, and let $U T M_{0}$ be its unit tangent bundle. Every map $U T M_{0} \rightarrow T^{n}$ is homotopic to a self-transversal smooth map which has to be an embedding since $n \geq 7$. Choose such an embedding $U T M_{0} \hookrightarrow T^{n}$ which induces an epimorphism of fundamental groups; let $M$ be the image of $U T M_{0}$ in $T^{n}$.

We consider flows on $M \simeq U T M_{0}$ preserving the Liouville measure. Each flow is generated by a divergence-free vector field on $M$. The rotation vector $\mathcal{R}(X) \in \mathbb{R}^{n} \cong H_{1}\left(T^{n} ; \mathbb{R}\right)$ of such a vector field $X$ is the average value of rotation vectors of its trajectories as curves in $T^{n}$. The map $X \rightarrow \mathcal{R}(X)$ is linear and the set of its values is the entire $\mathbb{R}^{n}$. Let $Y$ be a divergence-free vector field with $\mathcal{R}(Y)=v$.

Let $X_{0}$ be a vector field which generates the geodesic flow of our metric. It is skew-symmetric with respect to the relfection $w \mapsto-w$ of $U T M_{0}$, so $\mathcal{R}\left(X_{0}\right)=0$. Since the metric has negative curvature, its geodesic flow is an Anosov flow. Hence the vector field $X_{\varepsilon}:=X_{0}+\varepsilon Y$ still generates ergodic flow for $\varepsilon$ small enough. In particular, almost every trajectory generated by $X_{\varepsilon}$ has the rotation vector equal to $\mathcal{R}\left(X_{\varepsilon}\right)=\varepsilon v$. To complete the proof, consider the flow generated by $X_{\varepsilon} / \varepsilon$.

4.4. Let $n=8 r+6, v \in \mathbb{R}^{n}$ be a completely irratonal vector. Fix $M \subset T^{n}$ and a trajectory $\gamma: \mathbb{R} \rightarrow M$ of a smooth flow on $M$ constructed by Proposition 4.3. For each $x \in T^{n}$, identify the tangent space $T_{x} T^{n}$ with $\mathbb{R}^{n}$ in the standard way, and then consider the family $\left\{L_{x}=T_{x} M: x \in M\right\}$ of 3-dimensional linear subspaces of $\mathbb{R}^{n}$. Pick a $(8 r)$-dimensional linear subspace $L \subset \mathbb{R}^{n}$ which is transversal to 
this family, i. e. $L \cap L_{x}=\{0\}$ for all $x \in M$. Transversality implies that there exist $\varepsilon>0$, a tubular neighborhood $U \subset T^{n}$ of $M$, and a smooth retraction $p: U \rightarrow M$ such that $p(x+y)=x$ whenever $x \in M, y \in L$ and $|y|<\varepsilon$.

Let $\alpha \in \mathbb{R}$ be an approximable number. Using Proposition 4.2 , construct an $r$-stretching 1-periodic continuous curve $\gamma_{1}: \mathbb{R} \rightarrow L$ such that the map $f: \mathbb{R} \rightarrow L$ given by

$$
f(s)=\gamma_{1}(s+\alpha)-\gamma_{1}(s)
$$

is $C^{1}$-smooth. After a suitable homothety we may assume that $\left|\gamma_{1}(s)\right|<\varepsilon / 8$, and hence $|f(s)|<\varepsilon / 4$, for all $s \in \mathbb{R}$.

Denote $\sigma=1 / 3$. Fix a smooth function $\varphi:[0,1+\sigma] \rightarrow[0,1]$ such that $\varphi(t)=0$ for $t \in[0, \sigma]$ and $\varphi(t)=1$ for $t \in[1,1+\sigma]$. Define $\Gamma: \mathbb{R} \times[0,1+\sigma] \rightarrow T^{n+1} \times \mathbb{R}$ by

$$
\Gamma(s, t)=\left(\gamma(s+\alpha t)+\gamma_{1}(s)+f(s) \varphi(t),(s+\alpha t) \bmod 1, t\right) \in T^{n} \times S^{1} \times \mathbb{R} .
$$

4.5. Lemma. There exists $c>0$ such that

$$
\left|\Gamma(s, t)-\Gamma\left(s^{\prime}, t^{\prime}\right)\right| \geq c\left|\left(s-s^{\prime}\right) \bmod 1\right|^{1 / r}
$$

for all $s, s^{\prime} \in \mathbb{R}, t, t^{\prime} \in[0,1+\sigma]$.

Proof. For values of $\left|\left(s-s^{\prime}\right) \bmod 1\right|$ bounded away from zero, we may find a constant $c$ to satisfying (7) since

$$
\left|\Gamma(s, t)-\gamma_{2}\left(s^{\prime}, t^{\prime}\right)\right| \geq \min \{1,1 /|\alpha|\} \cdot\left|\left(s-s^{\prime}\right) \bmod 1\right|
$$

and the function $x \mapsto x^{1 / r} / x$ is bounded outside any neighborhood of zero. So it suffices to prove the statement of lemma when $\left|\left(s-s^{\prime}\right) \bmod 1\right|$ is sufficiently small.

First let $t^{\prime}=t=0$. We have

$$
\begin{aligned}
\left|\Gamma(s, 0)-\Gamma\left(s^{\prime}, 0\right)\right| & \geq\left|\gamma(s)+\gamma_{1}(s)-\gamma_{1}\left(s^{\prime}\right)-\gamma\left(s^{\prime}+\alpha t\right)\right| \\
& \geq \operatorname{dist}\left(M+\gamma_{1}(s)-\gamma_{1}\left(s^{\prime}\right), M\right) \geq c_{1}\left|\gamma_{1}(s)-\gamma_{1}\left(s^{\prime}\right)\right|
\end{aligned}
$$

since the vector $\gamma_{1}(s)-\gamma_{1}\left(s^{\prime}\right)$ lies in $L$ and its length is less than $\varepsilon$. Then (7) for $t^{\prime}=t=0$ follows from the fact that $\gamma_{1}$ is $r$-stretching and 1-periodic.

Since $\Gamma(s, t)-\Gamma(s, 0)$ is a $C^{1}$ (and hence Lipschitz) function of $(s, t)$, the estimate (7) for $t^{\prime}=t=0$ implies the same for $t^{\prime}=t \neq 0$. Then the complete statement follows since $\left|t-t^{\prime}\right| \leq\left|\Gamma(s, t)-\Gamma\left(s, t^{\prime}\right)\right| \leq C \cdot\left|t-t^{\prime}\right|$ for some constant $C$.

Define $\Gamma_{0}: \mathbb{R} \times[0,1+\sigma] \rightarrow T^{n+2}$ to be $\pi \circ \Gamma$ where $\pi: T^{n+1} \times \mathbb{R} \rightarrow T^{n+1} \times S^{1}=$ $T^{n+1}$ is the standard factorization. Note that for $t \in[0, \sigma]$,

$$
\begin{aligned}
\Gamma(s, t+1) & =\left(\gamma(s+\alpha+\alpha t)+\gamma_{1}(s)+f(s),(s+\alpha+\alpha t) \bmod 1, t+1\right) \\
& =\Gamma(s+\alpha, t)+(0,0,1) \in T^{n} \times S^{1} \times \mathbb{R},
\end{aligned}
$$


So

$$
\Gamma_{0}(s, t+1)=\Gamma_{0}(s+\alpha, t) \quad \text { for all } s \in \mathbb{R}, t \in[0, \sigma] .
$$

In other words, a piece of the surface $\Gamma_{0}(s, t)$ where $t \in[1,1+\sigma]$ matches the one where $t \in[0, \sigma]$, up to the parameter shift $s \mapsto s+\alpha$. $\Gamma$ has no other self-intersections since the last coordinate of $\Gamma_{0}(s, t)$ is $t \bmod 1$.

Let $w_{0}$ be the vector field along $\Gamma_{0}$ given by $w_{0}(s, t)=\frac{d}{d t} \Gamma_{0}(s, t)$. We are going to consider $w_{0}(s, t)$ as a function of point $\Gamma_{0}(s, t) \in T^{n+2}$. Though $w_{0}(s, t)$ is only $C^{1}$ as a function of $(s, t)$, it turns out to be smoother when viewed as a function on a subset of $T^{n+2}$.

4.6. Lemma. There exists a $C^{r-1}$-smooth vector field $W_{0}$ on $T^{n+2}$ such that $w_{0}=W_{0} \circ \Gamma_{0}$.

Proof. In view of $(8)$ it suffices to prove the same statement for a similar vector field along $\Gamma, w(s, t)=\frac{d}{d t} \Gamma(s, t)$. By Whitney's extension theorem ([F], pp. 225) it suffices to construct for each $(s, t) \in \mathbb{R} \times[0,1+\sigma]$ a smooth vector field $\varphi_{s, t}$ in a neighborhood of $\Gamma(s, t) \in T^{n+1} \times \mathbb{R}$ so that

$$
\left|w\left(\Gamma\left(s^{\prime}, t^{\prime}\right)\right)-\varphi_{s, t}\left(s^{\prime}, t^{\prime}\right)\right| \leq O\left(\left|\Gamma\left(s^{\prime}, t^{\prime}\right)-\Gamma(s, t)\right|^{r}\right), \quad\left(s^{\prime}, t^{\prime}\right) \rightarrow(s, t) .
$$

Recall that $\gamma$ is a trajectory of a smooth flow on $M \subset T^{n}$, so there is a smooth vector field $V$ on $M$ such that $\gamma^{\prime}(t)=V(\gamma(t))$ for all $t \in \mathbb{R}$. So

$$
w(s, t)=\left(\alpha V(\gamma(s+\alpha t))+f(s) g^{\prime}(t), \alpha, 1\right)
$$

is a $C^{1}$-smooth function of $(s, t)$. In particular,

$$
\left|w\left(t^{\prime}, \tau^{\prime}\right)-w\left(t, \tau^{\prime}\right)\right| \leq C\left|t^{\prime}-t\right|
$$

for some $C>0$. Let $U$ be a neighborhood of $M \subset T^{n}$ and $p: U \rightarrow M$ be a retraction as described in 4.4. Define $\varphi_{s, t}$ by

$$
\begin{aligned}
& \varphi_{s, t}(x, y, z)=\left(\alpha V(p(x-f(s) g(z)))+f(s) g^{\prime}(z), \alpha, 1\right) \\
& x \in U+f(s) g(z) \subset T^{n}, y \in S^{1}, z \in[0,1+\sigma] .
\end{aligned}
$$

Then $\varphi_{s, t}$ is a smooth function and $\varphi_{s, t}(\Gamma(s, t))=w(s, t)$ for all $s \in \mathbb{R}, t \in$ $[0,1+\sigma]$. Since $f$ is 1 -periodic, $\varphi_{s+m, t}=\varphi_{s, t}$ for any $m \in \mathbb{Z}$. Also, $\varphi_{s, t}$ does not depend on $t$ and $\varphi_{s, t}(x, y, z)$ is a $C^{1}$-smooth function of $(s, x, y, z)$. Then for any $m \in \mathbb{Z}$ we have

$$
\left|w\left(s^{\prime}, t^{\prime}\right)-\varphi_{s, t}\left(\Gamma\left(s^{\prime}, t^{\prime}\right)\right)\right|=\left|\varphi_{s^{\prime}, t^{\prime}}\left(\Gamma\left(s^{\prime}, t^{\prime}\right)\right)-\varphi_{s+m, t^{\prime}}\left(\Gamma\left(s^{\prime}, t^{\prime}\right)\right)\right| \leq C\left|s^{\prime}-s-m\right|
$$

for some constant $C$. Since $m$ is arbitrary,

$$
\left|w\left(s^{\prime}, t^{\prime}\right)-\varphi_{s, t}\left(\Gamma\left(s^{\prime}, t^{\prime}\right)\right)\right| \leq C\left|\left(s^{\prime}-s\right) \bmod 1\right|
$$


Using 4.5 we conclude that

$$
\left|w\left(s^{\prime}, t^{\prime}\right)-\varphi_{s, t}\left(\Gamma\left(s^{\prime}, t^{\prime}\right)\right)\right| \leq C\left|\Gamma\left(s^{\prime}, t^{\prime}\right)-\Gamma(s, t)\right|^{r}
$$

and the lemma follows.

Thus we have a flow on $T^{n+2}$ (generated by a $C^{r-1}$-smooth vector field $W_{0}$ ) such that all the curves $\Gamma_{0}^{s}:=\Gamma_{0}(s, \cdot):[0,1] \rightarrow T^{n+2}$ are intervals of its trajectories. Every curve $\Gamma_{0}^{s}$ joins the points $(\gamma(s), s, 0)$ and $(\gamma(s+\alpha), s+\alpha, 0)$ in $T^{n+2}$, and then the similar curve $\Gamma_{0}^{s+\alpha}$ forms the next piece of a trajectory. Clearly $\Gamma_{0}^{s}$ is homotopic to a curve $t \mapsto(\gamma(s+\alpha t), s+\alpha t, t) \in T^{n+2}$, so the rotation vector of the entire trajectory is equal to $(\alpha v, \alpha, 1)$, where $v \in \mathbb{R}^{n}$ is the rotation vector of $\gamma$ (see 4.4).

Since the last two coordinates of $w_{0}(\cdot)$ are the constants $\alpha$ and 1 , we may assume the same for $W_{0}(\cdot)$, i.e., $W_{0}(\cdot)=\left(W_{1}(\cdot), \alpha, 1\right)$ for some smooth $W_{1}: T^{n+2} \rightarrow \mathbb{R}^{n}$.

4.7. Let $\mathcal{M} \subset T^{n+2}$ be the image of $\Gamma_{0}$. Let $\tilde{W}_{0}$ and $\tilde{\mathcal{M}}$ be the lifts of $W_{0}$ and $\mathcal{M}$ from $T^{n+2}$ to $\mathbb{R}^{n+2}$. Both $\tilde{W}_{0}$ and $\tilde{\mathcal{M}}$ are $\mathbb{Z}^{n+2}$-invariant. Define two linear functions $L$ and $H$ on $\mathbb{R}^{n+2}$ by $L(x, y, z)=y-\alpha z$ and $H(x, y, z)=z$ for $x \in \mathbb{R}^{n}$, $y, z \in \mathbb{R}$. Here are the properties of $\tilde{W}_{0}$ and $\tilde{\mathcal{M}}$ that we will need:

(1) $\tilde{W}_{0}$ is a $C^{r-1}$-smooth vector field whose last two coordinates are $\alpha$ and 1. In particular, $L$ is constant and $H$ increases at the constant rate 1 under the flow generated by $\tilde{W}_{0}$.

(2) $\tilde{\mathcal{M}} \subset \mathbb{R}^{n+2}$ is invariant under the flow generated by $\tilde{W}_{0}$. At least one trajectory of $\tilde{W}_{0}$ lies in $\mathcal{M}$ and has rotation vector $(\alpha v, \alpha, 1)$.

(3) There exists $c>0$ such that

$$
|p-q| \geq c|L(p)-L(q)|^{1 / r} \quad \text { for all } x, y \in \tilde{\mathcal{M}} .
$$

These properties follow immediately from Lemma 4.5.

Let $g_{0}$ be a Riemannian metric on $\mathbb{R}^{n+2}$ which induces the standard flat metric on every hyperplane $\mathbb{R}^{n+1} \times\{$ const $\}$ and such that $\tilde{W}_{0}$ is a unit-length vector field orthogonal to these hyperplanes with respect to $g_{0}$. Clearly $g_{0}$ is uniquely determined, $C^{r-1}$-smooth, and $\mathbb{Z}^{n+2}$-periodic. Let $\rho_{0}$ denote the distance associated with $g_{0}$. Note that $L$ and $H$ are 1-Lipschitz functions with respect to $\rho_{0}$.

It is easy to construct a $C^{r-2}$-smooth $\mathbb{Z}^{n}$-periodic function $\varphi: \mathbb{R}^{n+2} \rightarrow \mathbb{R}$ such that $\left.\varphi\right|_{\tilde{\mathcal{M}}} \equiv 0$ and $\varphi(x) \geq \rho_{0}(x, \tilde{\mathcal{M}})^{r-1}$ for all $x \in \mathbb{R}^{n+2}$. Define a Riemannian structure $g$ on $\mathbb{R}^{n+2}$ by $g=g_{0} /(1+\varphi)^{2}$. $g_{0}$ is periodic and $C^{r-2}$-smooth.

Let $\rho$ be the metric determined by $g$ and let $\|\cdot\|$ be the stable norm of $\rho$. We will prove that the unit sphere of $\|\cdot\|$ is nonsmooth at the point $(\alpha v, \alpha, 1) \in \mathbb{R}^{n+2}$. First note that $\|(\alpha v, \alpha, 1)\| \leq 1$ by (2) above. 
4.8. Lemma. There exists an $\varepsilon>0$ such that

$$
\rho(p, q) \geq|H(p)-H(q)|+\varepsilon|L(p)-L(q)|
$$

whenever $H(p)-H(q)$ is integer, $p, q \in \mathbb{R}^{n+2}$.

Proof. Without loss of generality we may assume $0 \leq H(p)<1$ and $H(q) \geq$ $H(p)$. Since $\rho$ is a length metric, there is a sequence of points $p_{0}=p, p_{1}, \ldots$, $p_{N}=q$ such that $\rho(p, q)=\sum \rho\left(p_{i}, p_{i+1}\right)$ and for each $i=0, \ldots, N-1$ either $H\left(p_{i+1}\right)=H\left(p_{i}\right)$, or $H\left(p_{i+1}\right)=H\left(p_{i}\right)+1$ and a shortest curve joining $p_{i}$ and $p_{i+1}$ lies between the hyperplanes $H^{-1}\left(H\left(p_{i}\right)\right)$ and $H^{-1}\left(H\left(p_{i+1}\right)\right)$. It suffices to prove the desired estimate for each pair of points $\left(p_{i}, p_{i+1}\right)$ instead of $(p, q)$. In the case $H\left(p_{i}\right)=H\left(p_{i+1}\right)$ it holds with $\varepsilon=1$ because $L$ is 1 -Lipschitz with respect to $\rho$.

Let $H(q)=H(p)+1$ and let $\gamma$ be a $C^{1}$-smooth curve joining $p$ and $q$ and which lies between the hyperplanes $\{H=H(p)\}$ and $\{H=H(q)\}$. We assume that $\gamma:[0,1] \rightarrow\left(\mathbb{R}^{n+2}, \rho\right)$ is a constant speed parametrization. Let $\ell(\gamma)$ denote the length of $\gamma$ in $\left(\mathbb{R}^{n+2}, \rho\right)$. We have to check that $\ell(\gamma) \geq 1+\varepsilon|L(p)-L(q)|$ for some $\varepsilon>0$ independent of $\gamma, p$ and $q$.

Consider the map $f: \mathbb{R}^{n+1} \times[0,2] \rightarrow \mathbb{R}^{n+2}$ defined as follows: for $x \in \mathbb{R}^{n+1}$, $y \in[0,2], f(x, y)$ is the $y$-shift of the point $(x, 0) \in \mathbb{R}^{n+2}$ along the vector field $\tilde{W}_{0}$. Clearly $f$ preserves hyperplanes of the form $\{H=$ const $\}$, and $f^{-1}$ is a change of variables in $\mathbb{R}^{n+1} \times[0,2]$ which transforms $\tilde{W}_{0}$ into the $(n+2)^{n d}$ coordinate vector field. Since $f$ is a $\mathbb{Z}^{n+1}$-periodic $C^{1}$-smooth diffeomorphism, there are constants $c_{1}, c_{2}>0$ such that

$$
|x-y| / c_{1} \geq \rho_{0}(f(x), f(y)) \geq c_{1}|x-y| \quad \text { for all } x, y \in \mathbb{R}^{n+1} \times[0,2]
$$

and

$$
|x-y| \geq c_{2}|L \circ f(x)-L \circ f(y)|^{1 / r} \quad \text { whenever } x, y \in f^{-1}(\tilde{\mathcal{M}}) .
$$

Note that $L \circ f$ is the $(n+1)^{s t}$ coordinate function on $\mathbb{R}^{n+1} \times[0,2]$.

We may write $\gamma(t)=f\left(\gamma_{1}(t), \gamma_{2}(t)\right)$ for some $\gamma_{1}:[0,1] \rightarrow \mathbb{R}^{n+1}$ and $\gamma_{2}:[0,1] \rightarrow$ $[0,2]$. Clearly $g_{2}(t)=H \circ \gamma(t)$ for all $t$. Since the vector field $\tilde{W}_{0}$ is orthogonal to the hyperplanes $\{H=$ const $\}$ with respect to $\tilde{g}$, we have

$$
\left\|\gamma^{\prime}(t)\right\|_{g}=\left(1+\varphi(\gamma(t)) \cdot\left\|g^{\prime}(t)\right\|_{g_{0}} \geq\left(1+\varphi(\gamma(t)) \sqrt{c_{1}{\gamma_{1}^{\prime}}^{2}+{\gamma_{2}^{\prime}}^{2}} .\right.\right.
$$

Let $\delta=|L(p)-L(q)|$. Consider the following cases.

Case 1: $\delta \geq 2$. Then $\ell(\gamma) \geq \rho(p, q) \geq|L(p)-L(q)|=\delta \geq 1+\delta / 2$. In the remaining cases we suppose $\delta<2$.

Case 2: $\rho_{0}(p, \tilde{\mathcal{M}})$ and $\rho_{0}(q, \tilde{\mathcal{M}})$ are less than $c_{3} \delta^{1 / r}$ where $c_{3}=c_{1} c_{2} / 3$. It follows that $\gamma_{1}(0)$ and $\gamma_{1}(1)$ lie within $\left(\frac{1}{3} c_{2} \delta^{1 / r}\right)$-neighborhood of $f^{-1}(\tilde{\mathcal{M}})$ in $\mathbb{R}^{n+1} \times[0,2]$, so $\left|\gamma_{1}(0)-\gamma_{1}(1)\right| \geq \frac{1}{3} c_{2} \delta^{1 / r}$. Then

$$
\begin{aligned}
\ell(\gamma)^{2}=\int_{0}^{1}\left\|\gamma^{\prime}(t)\right\|_{g}^{2} & \geq \int_{0}^{1} c_{1} \gamma_{1}^{2}+\gamma_{2}^{\prime 2} \\
& \geq c_{1}\left|\gamma_{1}(1)-\gamma_{1}(0)\right|^{2}+\left|\gamma_{2}(1)-\gamma_{2}(0)\right|^{2} \geq 1+\frac{c_{1} c_{2}^{2}}{9} \delta^{2 / r} .
\end{aligned}
$$


Since $r \geq 2$, it follows that $\ell(\gamma) \geq 1+c_{4} \delta$ where $c_{4}$ can be easily expressed in terms of $c_{1}, c_{2}$ and the upper bound for $\delta$ (that is, 2).

Case 3: $\rho_{0}(p, \tilde{\mathcal{M}}) \geq c_{3} \delta^{1 / r}$ (or similar case with $q$ ). Let $\ell_{0}$ denote length with respect to $\rho_{0}$ and let $\gamma_{0}$ be the starting interval of $\gamma$ such that $\ell_{0}\left(\gamma_{0}\right)=\frac{1}{2} c_{3} d^{1 / r}$. Then $\gamma_{0}$ lies entirely outside the $\left(\frac{1}{2} c_{3} \delta^{1 / r}\right)$-neighborhood of $\mathcal{M}$ in $\left(\mathbb{R}^{n+2}, \rho_{0}\right)$. In this region, we have $\varphi \geq\left(\frac{1}{2} c_{3} \delta^{1 / r}\right)^{r-1}=c_{5} \delta^{1-1 / r}$. Therefore

$$
\ell(\gamma) \geq \ell_{0}(\gamma)+c_{5} \delta^{1-1 / r} \ell_{0}\left(\gamma_{0}\right) \geq 1+c_{6} \delta^{1 / r} \delta^{1-1 / r}=1+c_{6} \delta
$$

where $c_{6}=c_{3} c_{5} / 2$.

In all three cases, $\ell(\gamma) \geq 1+\varepsilon \delta$ for $\varepsilon=\min \left\{1 / 2, c_{4}, c_{6}\right\}$.

4.9. Corollary. $\|\cdot\| \geq H+\varepsilon|L|$.

Since $\|(\alpha v, \alpha, 1)\|=1$ and $L(\alpha v, \alpha, 1)=0$, both of the linear functions $H-\varepsilon L$ and $H+\varepsilon L$ support the unit ball of $\|\cdot\|$ at the point $(\alpha v, \alpha, 1)$. It follows that the unit sphere of $\|\cdot\|$ is nonsmooth at $(\alpha v, \alpha, 1)$.

\section{Acknowledgements}

The first author would like to thank V. Bangert and F. Nazarov for discussions which to some extent formed his vision of the problem. He also would like to thank A. Katok and N. Simanai for their patience while listening to the final version of the argument.

\section{References}

[AuD] S. Aubry and P. Y. LeDaeron, The descrete Frenchel-Kontorova madel and its exrtension I: Exact results for ground states., Physica 8D, 381-422.

[Ba1] V. Bangert, Minimal geodesics, Erg. Theory and Dyn. Syst. 10, 263-286.

[Ba2] Geodesic rays, Busemann functions and monotone twist maps, Calc. Var. $\mathbf{2}, 49-63$.

[Ba3] Mather sets for twist maps and geodesics on tori, In: Dynamics reported 1, $1-56$.

[Ba4] - Question, In: Address to Zurich IMC.

[Bu1] D. Burago, Periodic metrics, Representation theory and dynamical systems, Adv. Soviet Math., 9, New York, 1992, pp. 205-210.

[Bu2] _ Periodic metrics, in "Seminar on Dynamical Systems", Progress in nonlinear differential equations (H. Brezis, ed.), vol. 12, Birkhauser, 1994, pp. 90-95.

[BuI] D. Burago and S. Ivanov, Riemannian tori without conjugate points are flat, Geom. Funct. Anal. 4:3 (1994), 259-269.

[BuI2] - On asymptotic volume of tori, Geom. Funct. Anal. 5:5 (1995), 800-808.

[BuI3] , Isometric embeddings of Finsler manifolds, St. Petersburg Math. Journal 5:1 (1994), 159-169.

[CK] C. Croke and B. Kleiner, On tori without conjugate points, Bull. London Math. Soc. 22 (1990), 489-494.

[F] H. Federer, Geometric measure theory, Grund. Math. Wiss., 153.

[G] M. Gromov, Asymptotic invariants of infinite groups, Geometric group theory, Vol. 2, 1-295, London Math. Soc. Lecture Note Ser., 182, Cambridge Univ. Press, Cambridge, 1993. 
[GLP] M. Gromov, J. Lafontaine, P. Pansu, Structure metriques pour les varietés Riemanniennes, Metric structures for Riemann manifolds (J. Lafontaine and P. Pansu, ed.), Textes Mathèmatiques [Mathematical Texts], 1, CEDIC $\backslash$ Fernand Math, Paris, 1981.

[H] Jens Heber, On the geodesic flow of tori without conjugate points, Math. Z. 216 (1994), 209-216.

[Ka] M. Kac, Statistical independance in probability, Analysis and Number Theory, Carus Math. Monograph 12, Math. Asoc. Amer (1959).

[M1] John Mather, Variational construction of connecting orbits, Ann. Inst. Fourier (Grenoble) 43 (1993), 1349-1386.

[M2] _ Action minimizing invariant measures for positive definite Lagrangian systems, Math. Z. 207 (1991), 169-207.

[M3] Minimal measures, Comm. Math. Helv. 64, 375-394.

[M4] Differentiability of the minimal average action as a function of the rotation number, Bull. Soc. Bras. Mat. 21, 59-70.

[Na] Fedor Nazarov, Lecture given at Steklov institute, St. Petersburg.

Department of Mathematics, Pennsylvania State University, University Park, PA 16801 USA

E-mail address: burago@math.psu.edu

Steklov Institute for Mathematics, Fontanka 27, St. Petersburg 191028 RUSSiA

E-mail address: svivanov@pdmi.ras.ru

Department of Mathematics, University of Pennsylvania, Philadelphia, PA 191046395 USA

Current address: Department of Mathematics, University of Utah, Salt Lake City, UT 84112-0090 USA

E-mail address: bkleiner@math.upenn.edu 